\title{
A PRELIMINARY STUDY OF MORPHOMETRIC CHARACTERISTICS AND REPRODUCTIVE POTENTIAL OF THE FISH SPECIES COTTUS GOBIO LINNAEUS, 1758 ON SELECTED SITES FROM BOSNIA AND HERZEGOVINA
}

\author{
Selma Pilić1 ${ }^{*}$, Mahir Gajević1, Adi Vesnić1 ${ }^{1}$ Senad Šljuka ${ }^{1}$, Enad Korjenić1, Samir Đug ${ }^{1}$, \\ Aldijana Mušović1
}

${ }^{1}$ Faculty of Science, University of Sarajevo, Department of Biology, Sarajevo, Bosnia and Herzegovina

*Corresponding author

Selma Pilić, Asst. Prof.

Department of Biology, Faculty of

Science, University of Sarajevo,

Address: Zmaja od Bosne 33-35,

71000 Sarajevo, Bosnia and

Herzegovina

Phone: +38733723726

E-mail: selmam_@hotmail.com

ORCID: 0000-0002-0075-7885

Original Submission:

22 July 2021

Revised Submission:

16 September 2021

Accepted:

22 September 2021

\section{ABSTRACT}

The bullhead - Cottus gobio Linnaeus, 1758 is the only species from the Cottidae family in Bosnia and Herzegovina, and it inhabits the Una, Vrbas, Bosna and Drina River basins and the upper flow of the Neretva River. There are no reliable sources containing data on this species from those areas. Threats that are most commonly caused by chemical water pollution have led to local extinction, and the range of the species is currently fragmented with numerous isolated populations. The aim of this study was to assess the interpopulation variability of selected morphometric parameters and fertility of bullhead from different sites of the Una and Drina rivers in Bosnia and Herzegovina. The results of this study indicate that there is no statistically significant interpopulation variability of morphometric parameters and reproductive performance of Cottus gobio species in the waters of Bosnia and Herzegovina. A significant influence of ecological factors on the reproductive potential of the species Cottus gobio was recorded, especially in females, where a negative correlation with water temperature was established. Research on this scientifically neglected species should certainly be continued with the aim of better understanding and elucidating the causes of its geographical specificity and variability.

Key words: Bullhead, Una River, Drina River, morphology, fertility 


\section{INTRODUCTION}

Freshwater fish species from the Cottidae family have not been studied sufficiently, especially species of the genus Cottus. The Bullhead - Cottus gobio Linnaeus, 1758 is a relatively small fish species without special economic importance (Mann, 1971). It inhabits clean and cold freshwater ecosystems of Europe, and as a biotope prefers a rocky substrate poor in organic matter (Utzinger et al., 1998). In Europe, it is only absent in the Pyrenees, Apennines and the Southern Balkans, Ireland and northern England, Norway, Kola Peninsula and the White Sea Basin (Freyhof et al., 2005; Froese and Pauly, 2016). It mainly dominates in subtropical climates (distribution coordinates $70^{\circ} \mathrm{N} 40^{\circ} \mathrm{C}, 6^{\circ} \mathrm{W} \mathrm{60}{ }^{\circ} \mathrm{E}$ ) (Adamička, 1991).

Bull head is a nocturnal animal, most active during dusk, while during the day it hides in a rocky base. It is primarily a stationary species (Smyly, 1957), but it has been reported to migrate to tributaries (Crisp et al., 1984; Crisp and Mann, 1991) and the upper reaches of the river (Bless, 1990). It is stenovalent to temperature and oxygen, so it does not tolerate high temperatures and lack of oxygen (Hänfling et al., 2002). The density and biomass of the C. gobio species is higher in medium watercourses with high concentrations of dissolved calcium carbonate (Mills and Mann, 1983). They reach sexual maturity in the second year, with a total body length of $8 \mathrm{~cm}$. They spawn once a year when males change color (black with a white spot on the dorsal fin), while females increase body weight. Spawning takes place from February to April. Cannibalism on own eggs has been observed in the absence of other food sources (Sofradžija, 2009; Števove et al., 2019).

According to Sofradžija (2009), bullhead is the only species from the Cottidae family in Bosnia and Herzegovina, and inhabits the Una, Vrbas, Bosna and Drina River basins and the upper flow of the Neretva River. In $\mathrm{BiH}$, there are only studies based on the number and size of the population of C. gobio (Škrijelj et al., 2019).

Studies from other countries show that bullhead is a common species in those rivers with a general tendency to reduce habitats, most commonly caused by chemical water pollution (Bucher et al., 1992; Waterstraat, 1992; Utzinger et al., 1998; Hänfling et al., 2002; Knaepkens et al., 2004) by changing sediment in rivers and dam construction (Bless, 1981; Bless, 1990; Hofer and Bucher, 1991; Waterstraat, 1992; Jungwirth, 1996). These threats have led to the local extinctions, and the range of the species is currently fragmented with numerous isolated populations (Legalle et al., 2005; Van Liefferinge et al., 2005; Kanev and Uzunova, 2015).

Bullhead shows a high degree of morphological variation. It is generally characterized by a complete lateral line passing along the horizontal axis of the body and the absence of scales on the body. Morphological variations of Cottus gobio were analyzed in studies done by: Bãnãrescu, 1963; Scorepa, 1967; Witkowski, 1979; Frilund et al., 2009 and Uzunova et al., 2017.

Research on fish reproductive biology is crucial for assessing the fish stock potential and for the purpose of understanding species development (Dopeikar et al., 2015; Mousavi-Sabet et al., 2017). The sexual maturity of fish is closely related to the growth rate and body weight of a certain species, more precisely to its basic morphological characteristics. Fish fertility could be affected by various environmental factors, the impact of which varies depending on the species (Hänfling et al., 2002; Knaepkens et al., 2002; Lorenzoni et al., 
2018; Pilić et al., 2018). Factors such as size and age (Thorpe et al., 1984), Cottus survival strategy (Morita and Takashima, 1998), food availability and water temperature (Fleming and Gross, 1990; Ayoade, 2011) affect ichthyofauna fertility.

The aim of this study is to assess the interpopulation variability of selected morphometric parameters and fertility of Bullhead - Cottus gobio from different sites in Bosnia and Herzegovina. In accordance with the basic goal, the task was to establish a correlation between the analyzed parameters on the one hand, and the relationship with environmental factors on the other. It is important to note that there is no reference to this species in Bosnia and Herzegovina.

\section{MATERIAL AND METHODS}

Individuals of the Cottus gobio species were sampled in April 2019 at six sites: four on the Una River (Drobnica, Ripač, Kostela and Plandište) and two on the Drina River (Goražde-Osanica and Goradže-Vitkovići). Fishing was carried out with nets and predominantly by electrofishing (electric units "Honda" FEG 15,000, power $15 \mathrm{~kW}$ and "Honda" OHV 5.5, power $3.0 \mathrm{~kW}$ ).

The measurements of basic physico-chemical parameters relevant for biological parameters of water, were performed at the investigated sites. The water temperature, $\mathrm{pH}$ and the electrical conductivity of water were measured in the field using a multi-set apparatus for measuring chemical parameters of water (WTW Multi 3420 SET C) and the concentration of dissolved oxygen using the apparatus Oxi 3310 IDS Set 1.

In addition, data on the basic hydromorphological characteristics of the site were collected in the field.
The collected samples were fixed in $8 \%$ formaldehyde and transferred to the laboratory of the Center for Ichthyology and Fisheries (CIR) of the Faculty of Science, the University of Sarajevo. Individual identification was done in the field and laboratory according to the reference keys for this species (Vuković, 1977; Kottelat and Freyhof, 2007).

The measurements of the selected morphometric characteristics were carried out on the entire caught material (17): total body length, fork body length, standard body length, pre-anal length, dorsal fin base length, pre-ventral length, caudal peduncle length, head length, pre-orbital length, eye diameter, post-orbital length, pre-dorsal length, dorsal fin base length $\mathrm{d} 1$, dorsal fin base length $\mathrm{d} 2$, maximal body height and minimal body height. All these morphometric parameters were measured by using a Mitutoyo caliper. Total body weight (m) was determined using an analytical scale of the brand SOEHNLE Switzerland.

The sampled individuals of Bullhead were dissected for the purpose of sex determination and further analysis on the gonads. After dissection and removal of the digestive system, the gonads were observed bilaterally along the wall of the abdominal cavity. The same scale with an accuracy of two decimal places was used to determine the weight of the gonads.

Assessment of the general reproductive ability of individuals was performed by calculating the gonadosomatic index (GSI) (Jons and Miranda, 1997), using the formula (Bolger and Connolly, 1989):

$$
\mathrm{GSI}=\mathrm{Wg}(\mathrm{g}) / \mathrm{W}(\mathrm{g}) * 100
$$

Where:

$\mathrm{Wg}=$ weight of gonads

$\mathrm{W}=$ weight of fish 
Based on the obtained data, statistical analyzes and correlations were performed using the Statistics 8.0 software (C) Copyright StatSoft, Inc. 1984-2007). Morphometric measurements were performed in a larger number of replicates, and the results were presented as arithmetic means of several measurements with standard deviation. One way ANOVA was used to compare the variables, followed by the Post-hoc Newman-Keuls test (statistical significance level was $\mathrm{p}<0.05$ ). The Pearson product-moment correlation coefficient was used to examine the correlations between the variables, which is a measure of the linear correlation between the two variables tested.

\section{Ethical Approval and Consent to participate}

All applicable international, national, and/or institutional guidelines for the care and use of fish were followed by the authors. Fishing license issued by the Federal Ministry of Agriculture, Water Management and Forestry, Federation of Bosnia and Herzegovina, Number: 03-3-24/3$890 / 19$.

\section{RESULTS}

A total of 62 individuals of Cottus gobio were caught at six sites on the rivers Una and Drina (Drobnica, Ripač, Kostela, Plandište, Goražde-Osanica and Goradže-Vitkovići) (Figure 1, Table 1).

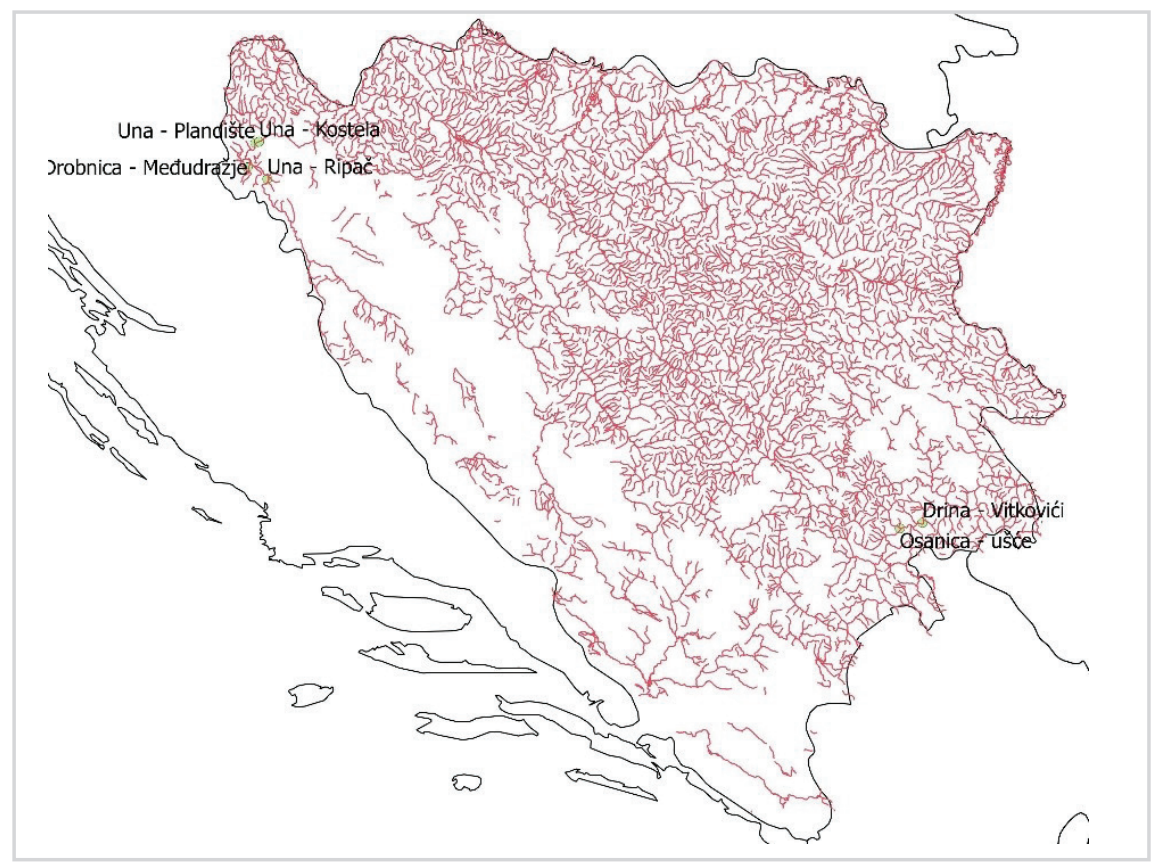

\section{Figure 1}

Map of research sites 
Table 1 Distribution of individuals and site characteristics

\begin{tabular}{|c|c|c|c|c|c|c|}
\hline \multirow[b]{2}{*}{ River/Site } & \multicolumn{2}{|c|}{ Drina } & \multicolumn{4}{|c|}{ Una } \\
\hline & $\begin{array}{l}\text { Goražde- } \\
\text { Vitkovići }\end{array}$ & $\begin{array}{l}\text { Goražde- } \\
\text { Osanica }\end{array}$ & Drobnica & Ripač & Plandište & Kostela \\
\hline Number of specimens $(\mathrm{N})$ & 12 & 6 & 12 & 10 & 10 & 12 \\
\hline Site length (m) & 200 & 100 & 100 & 200 & 200 & 200 \\
\hline Site width (m) & 61 & 3 & 3 & 52 & 125 & 35 \\
\hline Site area $\left(\mathrm{m}^{2}\right)$ & 12200 & 300 & 300 & 10400 & 25000 & 7000 \\
\hline $\begin{array}{l}\text { Average water column } \\
\text { depth }(\mathrm{m})\end{array}$ & 1.5 & 0.5 & 0.3 & 2.5 & 2.0 & 1.5 \\
\hline Elevation (m) & 343 & 370 & 262 & 229 & 218 & 201 \\
\hline Latitude $(\mathrm{N})$ & $43^{\circ} 37^{\prime} 38^{\prime \prime}$ & $43^{\circ} 36^{\prime} 36^{\prime \prime}$ & $44^{\circ} 46^{\prime} 12^{\prime \prime}$ & $44^{\circ} 45^{\prime} 57^{\prime \prime}$ & $44^{\circ} 48^{\prime} 57^{\prime \prime}$ & $44^{\circ} 53^{\prime} 4^{\prime \prime}$ \\
\hline Longitude (E) & $18^{\circ} 58^{\prime} 5^{\prime \prime}$ & $18^{\circ} 51^{\prime} 53^{\prime \prime}$ & $15^{\circ} 53^{\prime} 47^{\prime \prime}$ & $15^{\circ} 55^{\prime} 58^{\prime \prime}$ & $15^{\circ} 52^{\prime} 23^{\prime \prime}$ & $15^{\circ} 54^{\prime} 27^{\prime \prime}$ \\
\hline Air temperature $\left({ }^{\circ} \mathrm{C}\right)$ & 15.0 & 16.0 & 16.0 & 12.0 & 17.0 & 16.0 \\
\hline
\end{tabular}

The values of physico-chemical parameters measured at the sites on both rivers are mostly uniform (Table 2). However, the values of temperature and electrical conductivity of water are lower, and the values of dissolved $\mathrm{O}_{2}$ and $\mathrm{pH}$ of water are higher in the Drina River compared to the values measured in the Una River.

Table 2 Physico-chemical parameters of water at the investigated sites

\begin{tabular}{lcccccc}
\hline \multirow{2}{*}{ River/Site } & \multicolumn{3}{c}{ Drina } & \multicolumn{4}{c}{ Una } \\
\cline { 2 - 7 } & $\begin{array}{c}\text { Goražde- } \\
\text { Vitkovići }\end{array}$ & $\begin{array}{c}\text { Goražde- } \\
\text { Osanica }\end{array}$ & Drobnica & Ripač & Plandište & Kostela \\
\hline Water temperature $\left({ }^{\circ} \mathrm{C}\right)$ & 9.6 & 10.2 & 11.5 & 10.5 & 11.5 & 10.4 \\
\hline Dissolved oxygen $\left(\mathrm{mgO}_{2} / \mathrm{l}\right)$ & 11.7 & 11.8 & 11.2 & 11.5 & 10.8 & 11.6 \\
\hline $\mathrm{pH}$ of water & 8.0 & 8.1 & 7.8 & 7.6 & 7.6 & 7.8 \\
\hline $\begin{array}{l}\text { Electrical conductivity of } \\
\text { water }(\mu \mathrm{S} / \mathrm{cm})\end{array}$ & 295 & 275 & 410 & 450 & 420 & 440 \\
\hline
\end{tabular}

\section{Analysis of morphometric parameters}

All values of the measured morphometric parameters are presented as the mean value of the measurement \pm standard deviation. Statistically significant differences in the observed parameters are at the level of significance $\mathrm{p}>0.05$.

The values of the measured morphometric parameters of Cottus gobio male specimens $(\mathrm{N}=34)$ at individual research sites are shown in Table 3. These values at the sites of Osanica, Vitkovići, Plandište and Kostela are quite uniform. The highest values of morphometric parameters were recorded at the Drobnica site, while the lowest mean values of the measured morphometric parameters were recorded in 
individuals caught at the Ripač site. At the sites of Kostela, Ripač and Goražde-Vitkovići, larger variations of morphometric parameters were recorded in comparison with other sites (total body length, fork body length and standard length). The values of the length of the dorsal fin base (d1) and the length of the dorsal fin base (d2) of individuals from the Goražde -Vitkovići site have shown a large amplitude of variation.

The analyses of the One-Way ANOVA test have shown that there were no statistically significant differences between arithmetic means ( $\mathrm{p}>0.05)$ of male morphometric parameters.

Table 3 Values of morphometric parameters of males of the species Cottus gobio

\begin{tabular}{|c|c|c|c|c|c|c|}
\hline Site & $\begin{array}{c}\text { Goražde - } \\
\text { Osanica } \\
\text { N=2 }\end{array}$ & $\begin{array}{c}\text { Goražde - } \\
\text { Vitkovići } \\
\text { N=8 }\end{array}$ & $\begin{array}{l}\text { Drobnica } \\
\quad \mathrm{N}=2\end{array}$ & $\begin{array}{c}\text { Ripač } \\
\mathbf{N}=\mathbf{8}\end{array}$ & $\begin{array}{l}\text { Plandište } \\
\qquad N=6\end{array}$ & $\begin{array}{c}\text { Kostela } \\
\mathbf{N}=8\end{array}$ \\
\hline $\begin{array}{l}\text { Total body length } \\
(\mathrm{mm})\end{array}$ & $\begin{array}{c}89.69 \\
( \pm 3.10)\end{array}$ & $\begin{array}{l}83.66 \\
( \pm 6.64)\end{array}$ & $\begin{array}{l}113.04 \\
( \pm 2.08)\end{array}$ & $\begin{array}{c}74.63 \\
( \pm 5.48)\end{array}$ & $\begin{array}{l}86.98 \\
( \pm 3.22)\end{array}$ & $\begin{array}{c}85.86 \\
( \pm 11.48)\end{array}$ \\
\hline $\begin{array}{l}\text { Fork body length } \\
(\mathrm{mm})\end{array}$ & $\begin{array}{l}87.69 \\
( \pm 2.87)\end{array}$ & $\begin{array}{l}82.00 \\
( \pm 7.43)\end{array}$ & $\begin{array}{l}109.52 \\
( \pm 1.82)\end{array}$ & $\begin{array}{l}72.31 \\
( \pm 4.76)\end{array}$ & $\begin{array}{l}82.10 \\
( \pm 0.83)\end{array}$ & $\begin{array}{c}83.16 \\
( \pm 11.05)\end{array}$ \\
\hline $\begin{array}{l}\text { Standard body length } \\
(\mathrm{mm})\end{array}$ & $\begin{array}{l}76.84 \\
( \pm 2.00)\end{array}$ & $\begin{array}{l}71.73 \\
( \pm 5.69)\end{array}$ & $\begin{array}{c}95.89 \\
( \pm 1.98)\end{array}$ & $\begin{array}{c}62.09 \\
( \pm 5.49)\end{array}$ & $\begin{array}{l}71.35 \\
( \pm 1.52)\end{array}$ & $\begin{array}{c}73.37 \\
( \pm 10.07)\end{array}$ \\
\hline $\begin{array}{l}\text { Pre-anal length } \\
(\mathrm{mm})\end{array}$ & $\begin{array}{c}42.80 \\
( \pm 1.42)\end{array}$ & $\begin{array}{l}40.28 \\
( \pm 2.87)\end{array}$ & $\begin{array}{c}52.83 \\
( \pm 1.60)\end{array}$ & $\begin{array}{l}36.55 \\
( \pm 1.75)\end{array}$ & $\begin{array}{c}40.47 \\
( \pm 1.44)\end{array}$ & $\begin{array}{c}40.63 \\
( \pm 4.33)\end{array}$ \\
\hline $\begin{array}{l}\text { Dorsal fin base length } \\
(\mathrm{mm})\end{array}$ & $\begin{array}{l}22.58 \\
( \pm 1.20)\end{array}$ & $\begin{array}{l}21.09 \\
( \pm 1.40)\end{array}$ & $\begin{array}{c}26.81 \\
( \pm 1.00)\end{array}$ & $\begin{array}{c}16.99 \\
( \pm 2.42)\end{array}$ & $\begin{array}{c}20.22 \\
( \pm 2.80)\end{array}$ & $\begin{array}{c}20.86 \\
( \pm 3.47)\end{array}$ \\
\hline $\begin{array}{l}\text { Pre-ventral length } \\
(\mathrm{mm})\end{array}$ & $\begin{array}{c}22.17 \\
( \pm 0.80)\end{array}$ & $\begin{array}{c}19.41 \\
( \pm 1.19)\end{array}$ & $\begin{array}{l}24.50 \\
( \pm 0.98)\end{array}$ & $\begin{array}{c}18.63 \\
( \pm 1.23)\end{array}$ & $\begin{array}{c}19.57 \\
( \pm 1.30)\end{array}$ & $\begin{array}{c}20.85 \\
( \pm 2.58)\end{array}$ \\
\hline $\begin{array}{l}\text { Caudal peduncle length } \\
(\mathrm{mm})\end{array}$ & $\begin{array}{c}12.94 \\
( \pm 0.54)\end{array}$ & $\begin{array}{c}10.95 \\
( \pm 2.79)\end{array}$ & $\begin{array}{c}16.75 \\
( \pm 0.63)\end{array}$ & $\begin{array}{c}10.46 \\
( \pm 2.80)\end{array}$ & $\begin{array}{c}13.35 \\
( \pm 0.79)\end{array}$ & $\begin{array}{c}13.05 \\
( \pm 3.84)\end{array}$ \\
\hline $\begin{array}{l}\text { Head length }-\mathrm{HL} \\
(\mathrm{mm})\end{array}$ & $\begin{array}{c}23.00 \\
( \pm 0.72)\end{array}$ & $\begin{array}{c}22.80 \\
( \pm 1.38)\end{array}$ & $\begin{array}{l}25.58 \\
( \pm 0.90)\end{array}$ & $\begin{array}{c}18.83 \\
( \pm 1.29)\end{array}$ & $\begin{array}{c}20.85 \\
( \pm 0.92)\end{array}$ & $\begin{array}{c}21.15 \\
( \pm 2.77)\end{array}$ \\
\hline $\begin{array}{l}\text { Pre-orbital length } \\
(\mathrm{mm})\end{array}$ & $\begin{array}{c}6.61 \\
( \pm 0.21)\end{array}$ & $\begin{array}{c}6.73 \\
( \pm 1.78)\end{array}$ & $\begin{array}{c}8.42 \\
( \pm 0.18)\end{array}$ & $\begin{array}{c}6.17 \\
( \pm 0.35)\end{array}$ & $\begin{array}{c}6.15 \\
( \pm 0.29)\end{array}$ & $\begin{array}{c}6.71 \\
( \pm 0.88)\end{array}$ \\
\hline $\begin{array}{l}\text { Eye diameter } \\
(\mathrm{mm})\end{array}$ & $\begin{array}{c}5.25 \\
( \pm 0.27)\end{array}$ & $\begin{array}{c}5.03 \\
( \pm 0.39)\end{array}$ & $\begin{array}{c}5.13 \\
( \pm 0.67)\end{array}$ & $\begin{array}{c}4.41 \\
( \pm 0.87)\end{array}$ & $\begin{array}{c}4.88 \\
( \pm 0.35)\end{array}$ & $\begin{array}{c}5.23 \\
( \pm 0.43)\end{array}$ \\
\hline $\begin{array}{l}\text { Post-orbital length } \\
(\mathrm{mm})\end{array}$ & $\begin{array}{c}12.61 \\
( \pm 0.35)\end{array}$ & $\begin{array}{c}12.68 \\
( \pm 1.00)\end{array}$ & $\begin{array}{c}13.59 \\
( \pm 0.42)\end{array}$ & $\begin{array}{c}10.93 \\
( \pm 1.75)\end{array}$ & $\begin{array}{c}11.27 \\
( \pm 0.27)\end{array}$ & $\begin{array}{c}11.49 \\
( \pm 1.25)\end{array}$ \\
\hline $\begin{array}{l}\text { Pre-dorsal length } \\
(\mathrm{mm})\end{array}$ & $\begin{array}{l}26.98 \\
( \pm 0.92)\end{array}$ & $\begin{array}{l}27.87 \\
( \pm 1.81)\end{array}$ & $\begin{array}{c}32.94 \\
( \pm 0.78)\end{array}$ & $\begin{array}{c}23.67 \\
( \pm 1.92)\end{array}$ & $\begin{array}{c}25.34 \\
( \pm 0.87)\end{array}$ & $\begin{array}{c}27.11 \\
( \pm 3.53)\end{array}$ \\
\hline
\end{tabular}




\begin{tabular}{|c|c|c|c|c|c|c|}
\hline Site & $\begin{array}{c}\text { Goražde - } \\
\text { Osanica } \\
\text { N=2 }\end{array}$ & $\begin{array}{c}\text { Goražde - } \\
\text { Vitkovići } \\
\text { N=8 }\end{array}$ & $\begin{array}{c}\text { Drobnica } \\
\quad \mathrm{N}=2\end{array}$ & $\begin{array}{c}\text { Ripač } \\
\mathbf{N}=8\end{array}$ & $\begin{array}{l}\text { Plandište } \\
\qquad \mathrm{N}=6\end{array}$ & $\begin{array}{c}\text { Kostela } \\
\mathrm{N}=\mathbf{8}\end{array}$ \\
\hline $\begin{array}{l}\text { Dorsal fin base length } \\
\text { d1 }(\mathrm{mm})\end{array}$ & $\begin{array}{c}13.44 \\
( \pm 2.00)\end{array}$ & $\begin{array}{c}15.43 \\
( \pm 8.74)\end{array}$ & $\begin{array}{c}20.60 \\
( \pm 1.20)\end{array}$ & $\begin{array}{c}12.05 \\
( \pm 1.51)\end{array}$ & $\begin{array}{c}17.25 \\
( \pm 1.38)\end{array}$ & $\begin{array}{c}13.03 \\
( \pm 2.15)\end{array}$ \\
\hline $\begin{array}{l}\text { Dorsal fin base length } \\
\text { d2 }(\mathrm{mm})\end{array}$ & $\begin{array}{c}29.14 \\
( \pm 1.28)\end{array}$ & $\begin{array}{l}29.14 \\
( \pm 6.31)\end{array}$ & $\begin{array}{c}37.32 \\
( \pm 1.17)\end{array}$ & $\begin{array}{c}23.47 \\
( \pm 1.25)\end{array}$ & $\begin{array}{c}22.99 \\
( \pm 1.85)\end{array}$ & $\begin{array}{c}28.59 \\
( \pm 4.08)\end{array}$ \\
\hline $\begin{array}{l}\text { Maximal body height } \\
(\mathrm{mm})\end{array}$ & $\begin{array}{c}17.37 \\
( \pm 0.80)\end{array}$ & $\begin{array}{c}15.95 \\
( \pm 1.98)\end{array}$ & $\begin{array}{c}21.36 \\
( \pm 0.98)\end{array}$ & $\begin{array}{c}13.71 \\
( \pm 0.70)\end{array}$ & $\begin{array}{c}14.69 \\
( \pm 1.85)\end{array}$ & $\begin{array}{c}15.69 \\
( \pm 2.56)\end{array}$ \\
\hline $\begin{array}{l}\text { Minimal body height } \\
(\mathrm{mm})\end{array}$ & $\begin{array}{c}7.16 \\
( \pm 0.52)\end{array}$ & $\begin{array}{c}6.64 \\
( \pm 0.66)\end{array}$ & $\begin{array}{c}8.75 \\
( \pm 0.88)\end{array}$ & $\begin{array}{c}4.87 \\
( \pm 1.92)\end{array}$ & $\begin{array}{c}6.15 \\
( \pm 0.59)\end{array}$ & $\begin{array}{c}5.96 \\
( \pm 1.74)\end{array}$ \\
\hline Body weight (g) & $\begin{array}{c}13.2 \\
( \pm 0.90)\end{array}$ & $\begin{array}{c}8.40 \\
( \pm 2.04)\end{array}$ & $\begin{array}{c}19.6 \\
( \pm 0.58)\end{array}$ & $\begin{array}{c}5.56 \\
( \pm 1.30)\end{array}$ & $\begin{array}{c}7.87 \\
( \pm 1.05)\end{array}$ & $\begin{array}{c}9.80 \\
( \pm 4.99)\end{array}$ \\
\hline
\end{tabular}

*All values are presented as mean value \pm standard deviation

Comparative analysis of the morphometric parameters of females of Cottus gobio species $(\mathrm{N}=28)$ at the sites of Osanica, Vitkovići, Plandište and Drobnica showed quite uniform values (Table 4). The largest deviations were recorded at the site of Ripač, where the lowest mean values of all measured parameters were recorded in relation to other investigated sites. At the sites of Drobnica, Goražde-Osanica and Goražde-Vitkovići, larger variations of the minimum and maximum values of morphometric parameters were recorded: total body length, fork body length, standard body length, and total body weight.

The analyses of the One-Way ANOVA test have shown that there were no statistically significant differences between the arithmetic means of the measured morphometric parameters of females from the studied sites.

Table 4 Values of morphometric parameters of females of Cottus gobio species

\begin{tabular}{lcccccc}
\hline \multicolumn{1}{c}{ Site } & $\begin{array}{c}\text { Goražde }- \\
\text { Osanica } \mathbf{N = 4}\end{array}$ & $\begin{array}{c}\text { Goražde }- \\
\text { Vitkovići } \\
\mathbf{N = 4}\end{array}$ & $\begin{array}{c}\text { Drobnica } \\
\mathbf{N = 1 0}\end{array}$ & $\begin{array}{c}\text { Ripač } \\
\mathbf{N = \mathbf { 2 }}\end{array}$ & $\begin{array}{c}\text { Plandište } \\
\mathbf{N = 4}\end{array}$ & $\begin{array}{c}\text { Kostela } \\
\mathbf{N = 4}\end{array}$ \\
\hline $\begin{array}{l}\text { Total body length } \\
(\mathbf{m m})\end{array}$ & $\begin{array}{c}92.68 \\
( \pm 6.15)\end{array}$ & $\begin{array}{c}98.37 \\
( \pm 9.57)\end{array}$ & $\begin{array}{c}98.61 \\
( \pm 8.91)\end{array}$ & $\begin{array}{c}67.29 \\
( \pm 4.62)\end{array}$ & $\begin{array}{c}86.27 \\
( \pm 5.76)\end{array}$ & $\begin{array}{c}79.28 \\
( \pm 1.66)\end{array}$ \\
\hline $\begin{array}{l}\text { Fork body length } \\
(\mathbf{m m})\end{array}$ & 90.13 & 96.55 & 95.96 & 65.27 & 82.96 & 77.64 \\
$( \pm 6.97)$ & $( \pm 7.84)$ & $( \pm 8.81)$ & $( \pm 3.25)$ & $( \pm 3.40)$ & $( \pm 2.00)$ \\
\hline $\begin{array}{l}\text { Standard body length } \\
(\mathbf{m m})\end{array}$ & $\begin{array}{c}80.53 \\
( \pm 4.70)\end{array}$ & $\begin{array}{c}85.63 \\
( \pm 11.20)\end{array}$ & $\begin{array}{c}84.35 \\
( \pm 8.12)\end{array}$ & $\begin{array}{c}55.56 \\
( \pm 2.72)\end{array}$ & $\begin{array}{c}73.04 \\
( \pm 2.79)\end{array}$ & $\begin{array}{c}67.76 \\
( \pm 0.78)\end{array}$ \\
\hline
\end{tabular}




\begin{tabular}{|c|c|c|c|c|c|c|}
\hline Site & $\begin{array}{c}\text { Goražde } \\
\text { - Osanica } \\
\mathbf{N}=4 \\
\end{array}$ & $\begin{array}{c}\text { Goražde - } \\
\text { Vitkovići } \\
\mathrm{N}=4 \\
\end{array}$ & $\begin{array}{c}\text { Drobnica } \\
\mathbf{N}=10\end{array}$ & $\begin{array}{c}\text { Ripač } \\
\mathrm{N}=2\end{array}$ & $\begin{array}{c}\text { Plandišste } \\
\mathrm{N}=4\end{array}$ & $\begin{array}{c}\text { Kostela } \\
\mathrm{N}=4\end{array}$ \\
\hline $\begin{array}{l}\text { Pre-anal length } \\
(\mathrm{mm})\end{array}$ & $\begin{array}{l}45.20 \\
( \pm 4.05)\end{array}$ & $\begin{array}{c}49.53 \\
( \pm 9.04)\end{array}$ & $\begin{array}{c}47.82 \\
( \pm 4.08)\end{array}$ & $\begin{array}{c}31.61 \\
( \pm 1.87)\end{array}$ & $\begin{array}{c}41.01 \\
( \pm 2.97)\end{array}$ & $\begin{array}{c}39.35 \\
( \pm 0.97)\end{array}$ \\
\hline $\begin{array}{l}\text { Dorsal fin base length } \\
(\mathrm{mm})\end{array}$ & $\begin{array}{l}21.85 \\
( \pm 1.94)\end{array}$ & $\begin{array}{l}24.35 \\
( \pm 0.07)\end{array}$ & $\begin{array}{l}24.00 \\
( \pm 4.84)\end{array}$ & $\begin{array}{c}15.92 \\
( \pm 1.00)\end{array}$ & $\begin{array}{l}18.46 \\
( \pm 0.97)\end{array}$ & $\begin{array}{c}20.65 \\
( \pm 1.11)\end{array}$ \\
\hline $\begin{array}{l}\text { Pre-ventral length } \\
(\mathrm{mm})\end{array}$ & $\begin{array}{c}25.62 \\
( \pm 3.45)\end{array}$ & $\begin{array}{c}24.53 \\
( \pm 5.09)\end{array}$ & $\begin{array}{l}21.40 \\
( \pm 2.46)\end{array}$ & $\begin{array}{c}14.88 \\
( \pm 0.88)\end{array}$ & $\begin{array}{c}18.56 \\
( \pm 0.74)\end{array}$ & $\begin{array}{c}19.08 \\
( \pm 0.31)\end{array}$ \\
\hline $\begin{array}{l}\text { Caudal peduncle } \\
\text { length }(\mathrm{mm})\end{array}$ & $\begin{array}{c}16.93 \\
( \pm 1.22)\end{array}$ & $\begin{array}{c}18.02 \\
( \pm 4.48)\end{array}$ & $\begin{array}{l}13.76 \\
( \pm 2.38)\end{array}$ & $\begin{array}{c}8.30 \\
( \pm 0.61)\end{array}$ & $\begin{array}{c}12.47 \\
( \pm 1.65)\end{array}$ & $\begin{array}{l}10.63 \\
( \pm 0.42)\end{array}$ \\
\hline $\begin{array}{l}\text { Head length }-\mathrm{HL} \\
(\mathrm{mm})\end{array}$ & $\begin{array}{c}23.59 \\
( \pm 1.93)\end{array}$ & $\begin{array}{c}20.99 \\
( \pm 0.26)\end{array}$ & $\begin{array}{c}23.96 \\
( \pm 3.32)\end{array}$ & $\begin{array}{c}16.58 \\
( \pm 0.98)\end{array}$ & $\begin{array}{l}22.65 \\
( \pm 2.53)\end{array}$ & $\begin{array}{c}19.61 \\
( \pm 1.20)\end{array}$ \\
\hline $\begin{array}{l}\text { Pre-orbital length } \\
(\mathrm{mm})\end{array}$ & $\begin{array}{c}6.94 \\
( \pm 0.52)\end{array}$ & $\begin{array}{c}7.39 \\
( \pm 1.03)\end{array}$ & $\begin{array}{c}7.13 \\
( \pm 0.96)\end{array}$ & $\begin{array}{c}4.90 \\
( \pm 0.38)\end{array}$ & $\begin{array}{c}5.68 \\
( \pm 0.00)\end{array}$ & $\begin{array}{c}6.25 \\
( \pm 1.28)\end{array}$ \\
\hline Eye diameter $(\mathrm{mm})$ & $\begin{array}{c}5.50 \\
( \pm 0.58)\end{array}$ & $\begin{array}{c}5.29 \\
( \pm 0.40)\end{array}$ & $\begin{array}{c}5.21 \\
( \pm 0.68)\end{array}$ & $\begin{array}{c}3.83 \\
( \pm 0.00)\end{array}$ & $\begin{array}{c}5.08 \\
( \pm 0.00)\end{array}$ & $\begin{array}{c}5.29 \\
( \pm 0.01)\end{array}$ \\
\hline $\begin{array}{l}\text { Post-orbital length } \\
(\mathrm{mm})\end{array}$ & $\begin{array}{l}13.21 \\
( \pm 0.07)\end{array}$ & $\begin{array}{l}11.50 \\
( \pm 1.20)\end{array}$ & $\begin{array}{c}13.81 \\
( \pm 1.82)\end{array}$ & $\begin{array}{c}8.27 \\
( \pm 0.12)\end{array}$ & $\begin{array}{c}12.07 \\
( \pm 0.77)\end{array}$ & $\begin{array}{c}10.63 \\
( \pm 1.73)\end{array}$ \\
\hline $\begin{array}{l}\text { Pre-dorsal length } \\
(\mathrm{mm})\end{array}$ & $\begin{array}{c}27.02 \\
( \pm 0.89)\end{array}$ & $\begin{array}{c}27.84 \\
( \pm 1.79)\end{array}$ & $\begin{array}{c}28.97 \\
( \pm 3.59)\end{array}$ & $\begin{array}{c}20.95 \\
( \pm 1.00)\end{array}$ & $\begin{array}{l}27.05 \\
( \pm 2.21)\end{array}$ & $\begin{array}{c}24.97 \\
( \pm 0.31)\end{array}$ \\
\hline $\begin{array}{l}\text { Dorsal fin base length } \\
\text { d1 (mm) }\end{array}$ & $\begin{array}{c}13.57 \\
( \pm 1.78)\end{array}$ & $\begin{array}{c}14.68 \\
( \pm 1.76)\end{array}$ & $\begin{array}{c}16.53 \\
( \pm 2.01)\end{array}$ & $\begin{array}{c}11.37 \\
( \pm 0.43)\end{array}$ & $\begin{array}{c}14.54 \\
( \pm 0.62)\end{array}$ & $\begin{array}{l}11.25 \\
( \pm 2.70)\end{array}$ \\
\hline $\begin{array}{l}\text { Dorsal fin base length } \\
\text { d2 }(\mathrm{mm})\end{array}$ & $\begin{array}{l}30.70 \\
( \pm 0.07)\end{array}$ & $\begin{array}{c}31.61 \\
( \pm 5.03)\end{array}$ & $\begin{array}{l}31.90 \\
( \pm 3.57)\end{array}$ & $\begin{array}{l}21.96 \\
( \pm 0.22)\end{array}$ & $\begin{array}{l}29.60 \\
( \pm 2.22)\end{array}$ & $\begin{array}{c}25.99 \\
( \pm 2.92)\end{array}$ \\
\hline $\begin{array}{l}\text { Maximal body height } \\
(\mathrm{mm})\end{array}$ & $\begin{array}{l}18.65 \\
( \pm 2.13)\end{array}$ & $\begin{array}{l}16.73 \\
( \pm 2.60)\end{array}$ & $\begin{array}{l}18.27 \\
( \pm 2.45)\end{array}$ & $\begin{array}{c}12.90 \\
( \pm 1.08)\end{array}$ & $\begin{array}{l}14.95 \\
( \pm 0.98)\end{array}$ & $\begin{array}{c}14.01 \\
( \pm 1.13)\end{array}$ \\
\hline $\begin{array}{l}\text { Minimal body height } \\
(\mathrm{mm})\end{array}$ & $\begin{array}{c}7.41 \\
( \pm 0.70)\end{array}$ & $\begin{array}{c}6.85 \\
( \pm 1.42)\end{array}$ & $\begin{array}{c}7.74 \\
( \pm 1.15)\end{array}$ & $\begin{array}{c}5.05 \\
( \pm 0.38)\end{array}$ & $\begin{array}{c}5.69 \\
( \pm 0.00)\end{array}$ & $\begin{array}{c}6.18 \\
( \pm 0.53)\end{array}$ \\
\hline Body weight (g) & $\begin{array}{l}13.45 \\
( \pm 4.17)\end{array}$ & $\begin{array}{l}14.70 \\
( \pm 8.62)\end{array}$ & $\begin{array}{c}13.32 \\
( \pm 4.05)\end{array}$ & $\begin{array}{c}3.70 \\
( \pm 0.59)\end{array}$ & $\begin{array}{c}7.20 \\
( \pm 0.70)\end{array}$ & $\begin{array}{c}7.50 \\
( \pm 1.41)\end{array}$ \\
\hline
\end{tabular}

*All values are presented as mean value \pm standard deviation 
| PILIĆ ET AL. | POTENTIAL OF THE FISH SPECIES LOTUS GOBI LINNAEUS, 1758 ON SELECTED SITES FROM B\&H

301

Correlative temperature relations with selected morphometric parameters

Correlative relationships of the water temperature with selected morphometric parameters of males are presented in Figure 2. A positive correlation of total body length with temperature was recorded at all sites, with the value of the Pearson coefficient being $r=0.55$. The body length was positively correlated with the water temperature $(r=0.46)$ as well as weight $(\mathrm{r}=0.39)$.

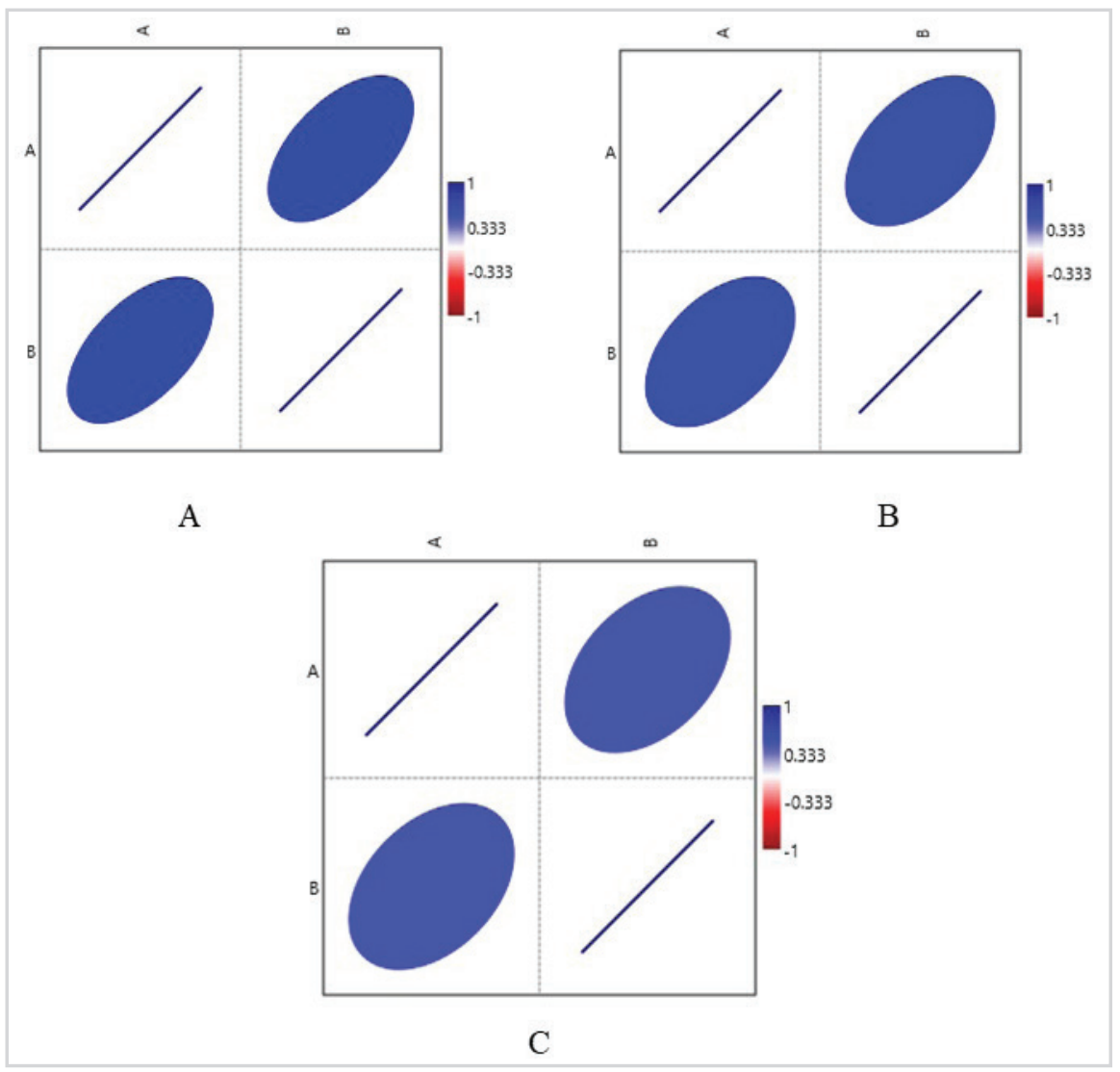

Figure 2

Correlative temperature relations with total body length (A), standard body length (B) and body weight (C) 
In the analysis of correlations between the water temperature of the investigated sites and selected morphometric parameters of Cottus gobio females, a negative correlation of the water temperature and standard body length was recorded (Figure 3). Total body weight showed a low degree of negative correlation with the water temperature, $\mathrm{r}=-0.26$.

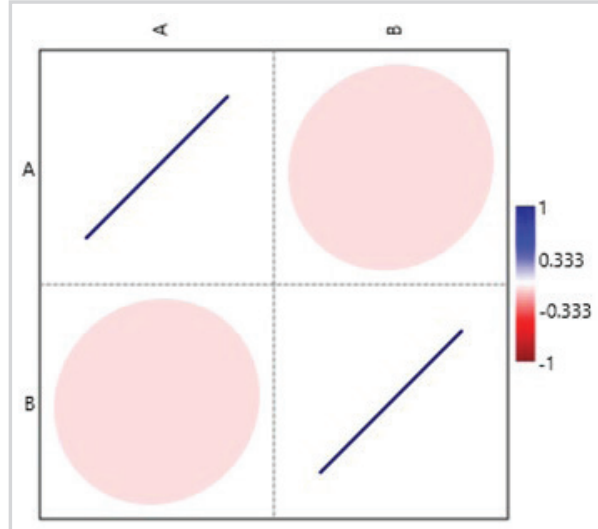

A

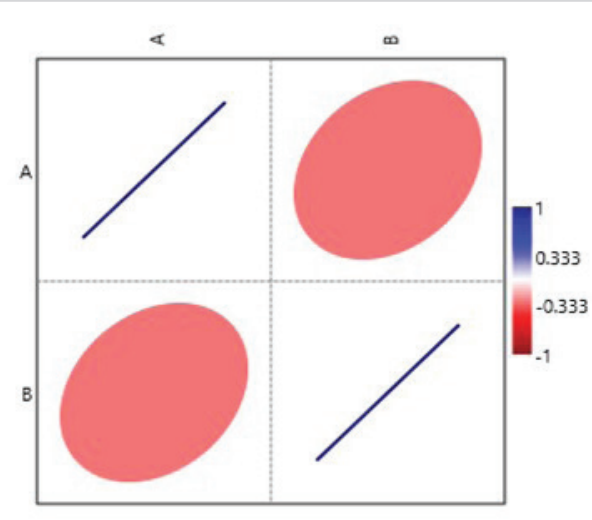

B

\section{Figure 3}

Correlative temperature relations with standard body length (A) and body weight (B)

\section{Analysis of gonadosomatic index values}

The values of the testicular weight of male Cottus gobio species sampled at the sites of Kostela, Plandište and Ripač are quite uniform. It is similar at the sites of Osanica and Vitkovići (Table 5). The highest value of the analyzed parameter was recorded at the site Drobnica, where the highest body weight of hunted individuals was recorded. The minimum value of the observed parameter was recorded at the Kostela site. The calculated values of GSI males range from $0.68 \%$ (Goražde Osanica) to $1.31 \%$ Ripač, which is also the highest recorded value of this parameter.

Table 5 Testicular weight and GSI male Cottus gobio species at six sites

\begin{tabular}{ccccccc}
\hline Site & $\begin{array}{c}\text { Goražde }- \\
\text { Osanica }\end{array}$ & $\begin{array}{c}\text { Goražde }- \\
\text { Vitkovići }\end{array}$ & Drobnica & Ripač & Plandište & Kostela \\
\hline Testicular weight $(\mathbf{g})$ & $\begin{array}{c}0.09 \\
( \pm 0.00)\end{array}$ & $\begin{array}{c}0.11 \\
( \pm 0.03)\end{array}$ & $\begin{array}{c}0.15 \\
( \pm 0.00)\end{array}$ & $\begin{array}{c}0.07 \\
( \pm 0.03)\end{array}$ & $\begin{array}{c}0.06 \\
( \pm 0.02)\end{array}$ & $\begin{array}{c}0.05 \\
( \pm 0.02)\end{array}$ \\
\hline GSI (\%) & $\begin{array}{c}0.68 \\
( \pm 0.00)\end{array}$ & $\begin{array}{c}1.05 \\
( \pm 0.15)\end{array}$ & $\begin{array}{c}0.76 \\
( \pm 0.00)\end{array}$ & $\begin{array}{c}1.31 \\
( \pm 0.25)\end{array}$ & $\begin{array}{c}0.71 \\
( \pm 0.25)\end{array}$ & $\begin{array}{c}0.74 \\
( \pm 0.04)\end{array}$ \\
\hline
\end{tabular}

* All values are presented as mean value \pm standard deviation

Statistically significant differences of the observed parameters at the level of significance $p>0,05$ 
The analysis of the One-Way ANOVA test showed that there were no statistically significant differences within the values of GSI individuals of males sampled from different sites.

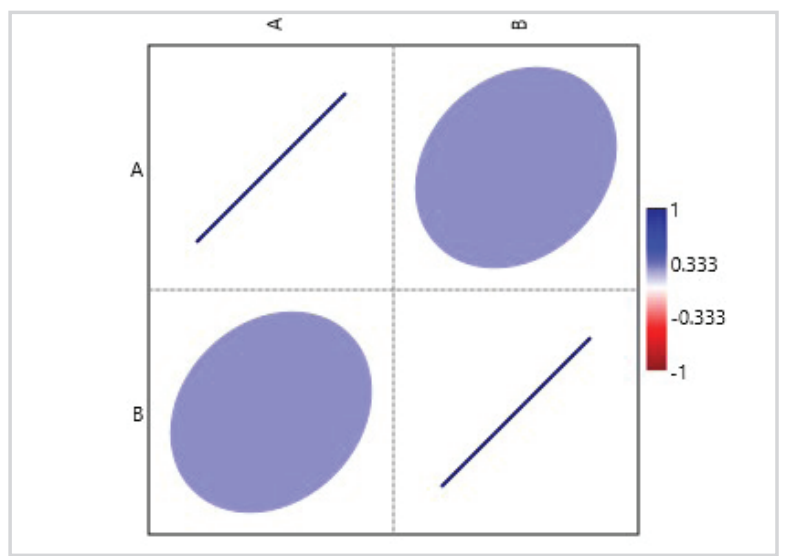

The correlation between the water temperature of the investigated fluids and the testis weight is presented in Figure 4. The value of the Pearson coefficient was $r=0.21$. GSI values also showed a positive correlation with the water temperature.

Figure 4 Correlative relationships of testicular weight (GSI) and water temperature

The weight of the ovaries at all the research sites shows a large amplitude of variation. The minimum values of the analyzed parameter were recorded at the site of Ripač (0.04 g), while the maximum values were observed at the site Goražde-Vitkovići $(0.40 \mathrm{~g})$. The highest value of gonadosomatic index (GSI) was recorded in the population of females sampled at the site Goražde-Vitkovići, and the lowest at the site Drobnica (Table 6).

Table 6 Values of gonadosomatic index of females at the researched sites

\begin{tabular}{ccccccc}
\hline Site & $\begin{array}{c}\text { Goražde- } \\
\text { Osanica }\end{array}$ & $\begin{array}{c}\text { Goražde }- \\
\text { Vitkovići }\end{array}$ & Drobnica & Ripač & Plandište & Kostela \\
\hline Ovarian weight $(\mathbf{g})$ & $\begin{array}{c}0.10 \\
( \pm 0.03)\end{array}$ & $\begin{array}{c}0.40 \\
( \pm 0.26)\end{array}$ & $\begin{array}{c}0.08 \\
( \pm 0.03)\end{array}$ & $\begin{array}{c}0.04 \\
( \pm 0.00)\end{array}$ & $\begin{array}{c}0.09 \\
( \pm 0.01)\end{array}$ & $\begin{array}{c}0.11 \\
( \pm 0.02)\end{array}$ \\
\hline GSI (\%) & $\begin{array}{c}0.77 \\
( \pm 0.02)\end{array}$ & $\begin{array}{c}2.69 \\
( \pm 0.16)\end{array}$ & $\begin{array}{c}0.72 \\
( \pm 0.50)\end{array}$ & $\begin{array}{c}1.08 \\
( \pm 0.00)\end{array}$ & $\begin{array}{c}1.26 \\
( \pm 0.32)\end{array}$ & $\begin{array}{c}1.52 \\
( \pm 0.07)\end{array}$ \\
\hline
\end{tabular}

* All values are presented as mean value \pm standard deviation

Statistically significant differences of the observed parameters at the level of significance $p>0,05$ 
The analysis of the One - Way ANOVA test showed that there was no statistically significant difference between the values of GSI females of the observed sites $(p>0.05)$.

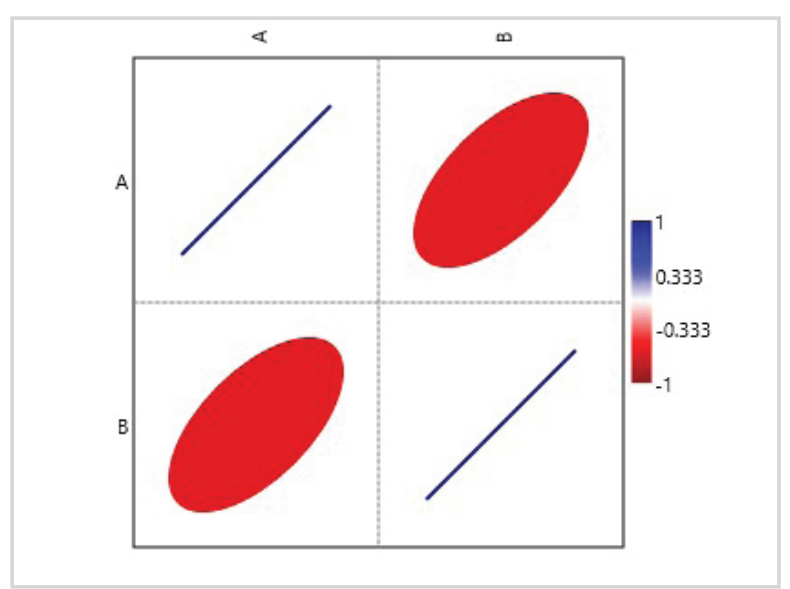

\section{DISCUSSION AND CONCLUSION}

The bullhead - Cottus gobio is a fish species of great conservation interest, listed in Annex II of EU Habitats Directive as "of community interest whose consideration requires the designation of special areas of conservation" (Lorenzoni et al., 2018). This fish species is threatened in many parts of its range by habitat alterations (Knaepkens et al., 2004), water pollution, water abstractions and predation by non-native species, such as brown trout (Salmo trutta Linnaeus, 1758) and restocked for angling purposes (Zerunian, 2002). There is no reference to this species in Bosnia and Herzegovina.

This research included the interpopulation variability of selected morphometric parameters and fertility of Bullhead from different sites in Bosnia and Herzegovina.
A negative correlation between the water temperature and the ovarian weight was determined, with the value of the coefficient $r=-$ 0.61 being recorded (Figure 5). The correlation between temperature and gonadosomatic index values generally had a negative trend.

\section{Figure 5}

Correlative relationships of ovarian weight (GSI) and water temperature

The values of physico-chemical parameters in the field of research are satisfactory within the physiological limits of this species (Adamička, 1991).

One of the most variable morphometric parameters of Cottus gobio species is total body length (Koli, 1969). In our study, the measured values of the monitored male parameters were evidently smaller compared to the values from the available literature. Muus and Dahlstrom (1968) state that the standard body length of male ranges from 100 $\mathrm{mm}$ to $140 \mathrm{~mm}$. Our results show lower values of the observed parameter with amplitude of variation from $75 \mathrm{~mm}$ to $103 \mathrm{~mm}$ were determined.

Lower head length values were also found compared to the values from the previous studies. Correlations between geographical regions and variations in morphometric parameters were recorded as well (Tsvetkov et al., 2001). 
Despite the variability of most morphological characteristics, some of them are relatively invariant and typical of different sites. For the purpose of comparison with similar studies, some values of morphometric parameters are expressed as a percentage (head length HL, standard body length SBL, maximum height/parameter value). The lowest head length in relation to SBL was found in females from the Drina River and amounted to $24.51 \%$. In the Drina River, the head length in relation to SBL in males on average varies from $29.93 \%$ to $31.78 \%$, while the mean values of the head length of individuals from the Una River basin range from $26.67 \%$ to $30.32 \%$. Head length in relation to SBL in females in the Drina River ranges on average from $24.51 \%$ to $29.29 \%$, while the values of this morphometric characteristic in the Una River basin are between $28.08 \%$ to $31.01 \%$. Data related to HL versus SBL were analyzed in individuals of Bullhead from the Chermosh River, whose length averaged 24.6\% SBL. In individuals from the rivers Severna Dvina, Po, Pechora, Volga and Zapadnaya Dvina, the head is larger and its length is on average from 27.1 to $27.9 \%$ of SBL.

The longest head lengths were recorded in samples from the Neva River systems, Lake Ladoga, and Aland Islands streams, and values ranged between 29.0-32.2\% SBL (Tsvetkov et al., 2001). Data on the length of the head of this species in different authors range from $27.4 \%$ SBL from the Pechora River (Korolev, 1991), 28.4-30.0\% SBL from the Danube River (Bãnãrescu, 1963), and 29.4-30, $3 \%$ SBL from the Oder, Elbe and Danube rivers (Witkowski, 1979).

The shortest anterior dorsal distance in the analyzed male individuals was recorded in the river Drobnica and amounted to $34.35 \% \mathrm{SBL}$, while the longest distance was found in individuals from the river Drina (38.85\% SBL). In the Drina River, this parameter in males ranged from 35.11 to $38.85 \%$ SBL on average. In the Una River basin, the antedorsal distance varied between 34.35 and $38.12 \%$ SBL in males. When it comes to the analysis of antedorsal distance in females in the Drina River, its values range from 32.51 to $33.55 \%$ SBL, and in the Una River basin between 34.34 and $37.7 \%$ SBL. The average value of antedorsal distance according to other authors is 28.1\% SBL in the Chermosh River, in the Neva River system, Lake Ladoga in the Aland Islands watercourses between 34.34 and $37.7 \%$ SBL, while these values are for specimens from the Danube, Po, Northern Dvina, Pechora, Volga and Zapadnaya Dvina between 31.2 and 34\% SBL (Tsvetkov et al., 2001), from the Pechora River 33.5\% SBL (Korolev, 1991), 30.7-34.0\% SBL in the Danube River (Bãnãrescu, 1963), 33.9-34.6\% $\mathrm{SBL}$ in the Elbe, Oder, Danube rivers (Witkowski, 1979). In contrast to these data, Witkowski (1979) cites values of $35.1 \%$ SBL for individuals with an incomplete lateral line in the rivers and lakes of Finland.

Certain differences were observed in both height and body shape in the analyzed individuals. The lowest average value of the maximum body height was recorded in males from the Una River at the Plandište site, and it was $20.58 \%$ SBL. In the Drina River, the values of this character in males ranged from 22.23 to $23.6 \% \mathrm{SBL}$, and in the Una River basin they ranged between 20.58 and $22.27 \%$ SBL. This character in females varied between 19.53 and $23.15 \%$ SBL in the Drina River, and in the Una River basin it was between 20.46 and $23.21 \%$ SBL. Values of this character in other studies range between $15.4 \% \mathrm{SBL}$ in the Pechora River to $20.9 \%$ SBL in the Neva River, Lake Ladoga, and the Aland Islands watercourses (Tsvetkov et al., 2001). 
In this study the task was to establish a correlation between the analyzed parameters and the relationship with environmental factors.

The physico-chemical parameters significantly affect the values of the character such as total body length, standard body length, body weight, etc. (Mortensen and Penczak, 1988). The maximum measured water temperature at the Plandište and Drobnica sites was $11.5^{\circ} \mathrm{C}$, while at the GoraždeVitkovići site the water temperature was $9.6{ }^{\circ} \mathrm{C}$, which is also the lowest water temperature at the selected sites. Since C. gobio dominates in a subtropical climate with a temperature range of $11{ }^{\circ} \mathrm{C}-16{ }^{\circ} \mathrm{C}$ (Adamička, 1991), the measured temperature values in our research are at the lower limit of this range or below it.

Males of most fish species mature sexually with lower body weight and one to two years before females. Compared to females, males spawn earlier and longer than females (Tyler et al., 1996; Bogut et al., 2006).

Ural and Özdemir (2011) found that changes in the physico-chemical properties of water (temperature, dissolved oxygen, $\mathrm{pH}$, nitrates, nitrites, ammonia, free $\mathrm{CO} 2$ and phosphates) result in slowed ovarian growth as well as an increased number of atretic follicles. Similar findings have been made by a number of other authors (Al Daham and Bhatti, 1979; Wootton, 1982; Okuzawa et al., 1989; Palmer et al., 1995; Pimpicka and Koryzno, 1995; Layl, 1998; Bardakci et al., 2000; Linares et al., 2002; Debarah et al., 2004; Kamanga et al., 2004; Ural and Özdemir, 2004), examining the impact of long-term changes in environmental factors on the gonadal growth and maturation follicle.

According to Dorts et al. (2011), it is observed that variation in temperature values significantly affects the gonadosomatic index value and ovulation in females, and the secretion of sex hormones in males. The results of this study, especially the correlations between gonadal weight and the water temperature, indicate that global warming affects the reproduction of Cottus gobio species, which is confirmed by the results of our study.

Females at a water temperature of $6-10{ }^{\circ} \mathrm{C}$ had a mean GSI value of $22.5 \pm 1.2 \%$ at which ovulation occurred. Females at a slightly elevated temperature (10-14 ${ }^{\circ} \mathrm{C}$ ) had a GSI value of $15.3 \pm 1.6 \%$, and ovulation also occurred. The group of females that were in water at a temperature of $14-18{ }^{\circ} \mathrm{C}$ had a significantly reduced GSI $(4.1 \pm 0.7 \%)$. and ovulation was completely absent. At the elevated temperature, the gonadosomatic index in males ranged from 0.1 to $2.9 \%$, indicating that these temperature oscillations had no effect on the change in GSI values. However, it has been observed that sex hormones cease to be secreted at elevated temperatures (Dorts et al., 2011).

Based on the conducted studies, it is evident that physico-chemical factors significantly affect the growth of the gonads, which corresponds to the results of our research. According to some research, fertility variations may also be due to different amounts of food in the environment (Fleming and Gross, 1990; Ayoade, 2011).

Comparing the results of our research with the results of other analyzed studies, it is evident that the GSI values are much lower at the same temperature values. The reason for this can certainly be the different age structure of individuals. Larger deviations were observed in females, which also coincides with the results of previous studies.

The results of this study indicate that there is no statistically significant interpopulation variability of the morphometric parameters and reproductive performance of Cottus gobio species 
in the waters of Bosnia and Herzegovina. The identified differences in the mean values of the monitored parameters can be related to different micro-climatic environmental conditions and food availability, which is conditioned by the sampling sites themselves. A large variability of morphometric parameters was found compared to previous research, which is undoubtedly related to the different geographical area of the studied population of Bullhead and all the conditions related to it. Significant influence of ecological factors on the reproductive potential of the species Cottus gobio was recorded, especially in females, where a negative correlation with water temperature was found. Research on this scientifically neglected species should certainly be continued with the aim of better understanding and elucidating the causes of its geographical specificity and variability.

\section{ACKNOWLEDGEMENTS}

This research did not receive any specific grant from funding agencies in the public, commercial, or not-for-profit sectors.

\section{CONFLICT OF INTEREST}

The authors declared that there is no conflict of interest.

\section{REFERENCES}

Adamička P. 1991. Schicksaleinerdurchschnittlichen Koppe (Cottus gobio L.) im Lunzer Seebach. Österreichs Fischerei, 44, 162-4.

Al Daham NK, Bhatti MN. 1979. Annual changes in the ovarian of the freswater teleost. Barbusluteus (Heckel) from Southern Iraq. J Fish Biol, 14, 381-7.

Ayoade AA. 2011. Length-weight relationship and diet of African carp Labeoogunensis (Boulenger 1910) in Asejire Lake Southwestern Nigeria. Journal of fisheries and Aquatic Science, 6(4), 472-8.

Bãnãrescu P. 1963. Date biometrice sisistematice a supragenului Cottus (Pisces Cottidae) in RPR (Biometrical and systermatical notes on the genus Cottus (Pisces Cottidae) in the Rumanian peoples Republic). Soc St Nat Geogr RPR Com Zool, 2, 119-34.

Bardakci F, Ozansoy U, Koptagel E. 2000. A comparison of oogenesis under constant and fluctuating temperatures in Doctor fish Garrarufa (Heckel 1843). J Fish Biol, 5, 1-12.

Bless R. (1981.) UntersuchungenzumEinfluss von gewisserbaulichen Massnahmen auf die Fischfauna in Mittelgebirgsbichen. Natur und Landschufi, 56, 243-52.

Bless R. 1990. Die Bedeutung von gewasserbaulichenHin dernissenimRaum-Zeit-System der Groppe(CottusgobioL). Natur Und Land rchafi, 65, 581-5.
Bogut I, Novoselić D, Paličević J. 2006. Biologijariba. Sveučilište JJ Strossmayera Osijek i Sveučilište u Mostaru, Osijek.

Bolger J, Connolly PL. 1989. The selection of suitable indices for the measurement and analysis of fish condition. J Fish Biol, 34, 171-82.

Bucher F, Hofer R, Salvenmoser W. 1992. Effects of treated paper mill effluents on hepatic morphology in male bullhead (CottusgobioL). Archives of Environmental Contamination and Toxicology, 23, 410-9.

Crisp DT, Mann RHK. 1991. Effects ofimpoundment on populations of bullhead Coitus gobioL and minnow Phoxinusphoxinus (L) in the Basin of Cow Green Reservoir. J Fish Biol, 38, 731-40.

Crisp DT, Mann RHK, Cubby PR. 1984.Effects of impoundment upon fish populations in afferent streams at Cow Green Reservoir. J Appl Ecol, 21, 739-56.

Debarah JM, Robiohavd D, Berlinsky L. 2004. The effects of phototermal manipulation on reproductive development in female haddock Melanogrammusaeglefinus L. Aquac Res, 35(5), 465-72.

Dopeikar H, Keivany Y, Shadkhast M. 2015. Reproductive biology and gonad histology of the kurabarbel Barbuslacerta (Cyprinidae) in Bibi-Sayyedan River Tigris basin Iran NorthWestern. J Zool, 11, 163-70.

Dorts J, Grenouillet G, Douxfils J, Mandiki NMS, Milla S, Silvestre F, Kestemont P. 2011. Evidence that elevated water temperature affects the reproductive physology of etve 
European bullhead Cottus gobio. Fish Physiol Biochem, 8, $30-45$.

Fleming IA, Gross MR. 1990. Latitudinal clines: a trade-off between egg number and size in Pacific salmon. Ecol, 71(1), 1-11.

Freyhof J, Kottelat M, Nolte A. 2005. Taxonomic diversity of European Cottus with description of eight new species (Teleostei: Cottidae). Ichthyol Explor Freshw, 16, 107-72.

Frilund GE, Koksvik J, Rikstad A, Berger HM. 2009. Cottus gobio (Linnaeus, 1758), a new fish-species in Nord-Trøndelag County, Norway. Fauna norvegica, 29, 55-60.

Froese F, Pauly D. 2016. Fish Base. http://www.fishbase.org version (01/2016), (accessed 23.04.2021).

Hänfling B, Hellemans B, Volckaert FAM, Carvalho GR. 2002. Late glacial history of the cold-adapted freshwater fish Cottus gobio revealed by microsatellites. Mol Ecol, 11, 1717-29.

Hofer R, Bucher F. 1991. ZurBiologie und Gefahrdung der KoppeOsterreichs. Fischerei, 44, 158-61.

Jons GD, Miranda LE. 1997. Ovarian weight as an index of fecundity maturity and spawning periodicity. J Fish Biol, 50, 150-6.

Jungwirth M. 1996. Bypass channels a t weirs as appropriate aids for fish migration in rithoral rivers. Regulated Riaers Research and Management,12, 483-92.

Kanev E, Uzunova E. 2015. Effects of habitat fragmentation on current distribution of the genus Cottus (Cottidae) in Bulgaria, „Fish Passage 2015 International conference on river connectivity best practices and innovations", 22-24 June. Groningen, Holland.

Kamanga LJ, Kaunda JP, Mtimuni AO, Maluwa WM. 2004. Effect of temperature on ovocyte Oreochromis karongae (Trewavas 1941). J ApplIchthyol, 20(2), 139-45.

Knaepkens G, Knapen D, Hänfling B, Verheyen E, Eens M. 2002. Genetic diversity and condition factor: a significant relationship in Flemish but not in German populations of the European bullhead (Cottus gobio L.). Heredity, 89, 280-7.

Knaepkens G, Bruyndoncx L, Coeck J, Eens M. 2004. Spawning habitat enhancement in the European bullhead (Cottus gobio) an endangered freshwater fish in degraded lowland rivers. Biodivers Conserv, 13, 2443-52.

Koli L. 1969. Geographical variation of Cottus gobio L. (Pisces Cottidae) in Northen Europe. Ann ZoolFenn, 6, 353-90.

Korolev VV. 1991. Common bullhead Cottus gobio from the drainage of Upper reaches of Pechora Voprosy. Ikhtiologii, 31(1), 162-5.
Kottelat M, Freyhof J. 2007. Handbook of European freshwater fishes. Publications Kottelat, Cornol, Switzerland, p. 646.

Layl AL. 1998. Effect of environmental pollution by crude oil on the ovary of the fish (Oreochromis niloricus). J Union Arab Biol, 10, 141-8.

Legalle M, Santoul F, Figuerola J, Mastrorillo S, Céréghino R. 2005. Factors influencing the spatial distribution patterns of the bullhead (Cottus gobio L Teleostei Cottidae): a multiscale study. Biodivers Conserv, 14, 1319-34.

Linares J, Eerennaam VJP, Doroshow SI. 2002. Ultrastructural and histological observation on temperatureinduced follicular ovarian atresia in the white sturgeon. J Fish Biol, 62(2), 253-75.

Lorenzoni M, Carosi A, Giovannotti M, Porta GL, Splendiani A, Barucchi VC. 2018. Population status of the native Cottus gobio after removal of the alien Salmo trutta: a case-study in two Mediterranean streams (Italy). Knowl Manag Aquat Ecosyst, 419-22.

Mann RHK. 1971. The populations growth and production of fish in four small streams in southern England. J Anim Eco, 40, 155-90.

Morita K, Takashima Y. 1998. Effect of female size on fecundity and egg size in white-spotted charr: comparison between sea-run and resident forms. J Fish Biol, 53(5), 1140-2.

Mortensen E, Penczak T. 1988. Populations growth biomass and production of fish in a small stream in northwest Polan. Ekologia Polska, 36, 445-8.

Mousavi-Sabet H, Abdollahpour S, Vatandoust S, FaghanILangroudi H, Salehi-Farsani A, Salehi M, et al. 2017. Reproductive biology of Alburnus mossulensis (Teleostei: Cyprinidae) in Gamasiab River western Iran. Iran J Ichthyol, 4(2), 171-80.

Muus BJ, Dahlström P. 1968. Süsswasserfische BLV. Verlagsgesellschaft.

Mills C, Mann RHK. 1983. The bullhead Cottus gobio a versatile and successful fish. Rep Fresh Biol Assoc, 51, 76-88.

Okuzawa K, Furukawa K, Aida K, Hanyu I. 1989. Effect od photoperiod and temperature on gonadal maturation and plasma steroid and gonadotropin levels in a cyprinid fish honmoroko Gnathopogen cacrulescens. Gen Comp Endocrinol, 75, 139-47.

Palmer EE, Sorenson PW, Adelman IR. 1995. A histological study of seasonal ovarian development in fresh water drum in the red lakes Minnesota. J Fish Biol, 47, 199-210. 
Pilić S, Mlaćo N, Katica A, Katica V, Mujezinović I, Katica J. 2018. Histological characteristic features of the rainbow trout Oncorhynchus mykiss ovaries (Walbaum 1792) grown in various microambient conditions. Veterinary Journal of Republic of Srpska, 18(2), 326-341.

Pimpicka E, Koryzno A. 1995. Ovary development in juvenile tench Tinca tinca reared in different thermal conditions Polskie Archiwum. Hydrobiologii, 42, 75-83.

Scorepa V.1967.ZurSistematik der Bomishen West-bropen (Cottus gobio L.) Vest CsSpol. Zool, 31(3), 260-73.

Smyly WJP. 1957. The life-history of the bullhead or miller's thumb (Cottus gobio L.). Proceedings of the Zoological Society of London, 128, 431-53.

Sofradžija A. 2009. Slatkovodne ribe Bosne I Hercegovine. Vijeće Kongresa bošnjačkih intelektualaca, Sarajevo. p. 176.

Števove B, Babel'ová M, Haruštiaková D, Kováč V. 2019. When a river is affected by a reservoir: Trophic interactions and flexibility in feeding strategies of alpine bullhead (Cottus poecilopus) and European bullhead (Cottus gobio). Science of the Total Environment 651, 1903-12.

Škrijelj R, Đug S, Korjenić E, Drešković N, Vesnić A, Gajević M, Šljuka S, Mušović A, Pilić S. 2019. Ihtiološka istraživanja sliva rijeke Save u Federaciji Bosne i Hercegovine. Agencija za vodno područje rijeke Save. Centar za ihtiologiju i ribarstvo, Prirodno-matematilčki fakultet Univerziteta u Sarajevu, Sarajevo. p. 86.

Thorpe JE, Miles MS, Keay DS. 1984. Developmental rate fecundity and egg size in Atlantic salmon Salmo salar L. Aquacul, 43(1-3), 289-305.

Tyler CR, Pottinger TG, Santos E, Sumpter JP, Price SA, Brooks S, et al. 1996. Mechanisms Controlling Egg Size and Number in the Rainbow Trout Oncorhynchus mykiss. Biol Reprod, 54, 8-15.

Tsvetkov IB, Sideleva VG, Bogutskaya NG. 2001. Morphological variation in bullhead Cottus gobio Linnaeus 1758 (Cottidae) new contributions to freshwater fish research St Petersburg. Proceedings of the zoological institute, 287, 121-30.
Ural M, Özdemir Y. 2004. The morphological characteristic and ovarium histological structure of freswater chub ( $L$ cephalus) in the Keban Dam Lake University of Firat. Journal of Science Engineer, 12(1), 313-21.

Ural M, Özdemir Y. 2011. Water Quality from Koçkale and Pertek Regions of Keban Dam Lake (Elazığ/Turkey) on Ovarium Tissue of Capoeta trutta (Heckel 1843) Histopathological Effect. Aust J Basic Appl Sci, 5(8), 1096-1105

Utzinger J, Roth C, Peter A. 1998. Effects of environmental parameters on the distribution of bullhead Cottus gobio with particular consideration of the effects of obstructions. J Appl Ecol, 35, 882-92.

Uzunova EP, Kanev EK, Stefanov T. 2017. Spatial Variation in the Abundance and Population Structure of Bullhead Cottus gobio L., 1758 (Actinopterygii: Cottidae) from the Iskar River Basin (Danube River Drainage, Bulgaria): Implications for Monitoring and Conservation. Acta Zzoological Bulgarica, 69 (3), 393-404.

Van Liefferinge C, Seeuws P, Meire P, Verheyen R F. 2005. Microhabitat use and preferences of the endangered Cottus gobio in the River Voer Belgium. J Fish Biol, 67, 897-909.

Vuković T. 1977. Ribe Bosne i Hercegovine: ključ za određivanje. Sarajevo, Svjetlost.

Waterstraat A. 1992. Investigations on the ecology of Cottus gobio L. and other fish species from two lowland streams of northern Germany. Limnologica, 22, 137-49.

Witkowski A. 1979. A taxonomic study on fresh water sculpins of the genus Cottus Linnaeus 1758 (Cottus gobio L. and Cottus poecilopus Heck) in Poland. Acta Universitatis Wratislaviensis, 458, 1-44.

Wootton RJ. 1982. Environmental factors in fish reproducton. In Proc Int Symposium on Reproductive Physiology of Fish DUDOC Wageningen The Netherlands, 210-19.

Zerunian S. 2002. Iconografia deipescidelleacque interne d'Italia (Iconography of Italian inland water fishes). Modena: Tipolitog-rafia FG, 259. 


\section{PRELIMINARNO PROUČAVANJE MORFOMETRIJSKIH KARAKTERISTIKA I REPRODUKTIVNOG POTENCIJALA RIBE COTTUS GOBIO LINNAEUS, 1758 NA ODABRANIM LOKACIJAMA IZ BOSNE I HERCEGOVINE}

\section{SAŽETAK}

Peš - Cottus gobio Linnaeus, 1758 predstavlja jedinu vrstu iz porodice Cottidae u Bosni i Hercegovini i naseljava riječne bazene rijeke Une, Vrbasa, Bosne i Drine te gornji tok rijeke Neretve. Iz navedenih područja nema pouzdanih podataka o ovoj vrsti. Prijetnje koje su najčešće posljedica hemijskog zagađenja vode su dovele do lokalnog izumiranja, a vrsta je trenutno fragmentirana u mnogobrojne izolirane populacije. Cilj istraživanja je procijeniti interpopulacijsku varijabilnost pojedinih morfometrijskih parametara i fertilitet peša koji potječe sa različitih lokacija iz rijeka Une i Drine u Bosni i Hercegovini. Rezultati našeg istraživanja pokazaju da ne postoji statistički signifikantna interpopulacijska varijabilnost morfometrijskih parametara i reproduktivnih performansi vrste Cottus gobio u vodama Bosne i Hercegovine. Zabilježen je značajan utjecaj koji ekološki faktori imaju na reproduktivni potencijal vrste Cottus gobio, posebice kod ženki, kao i negativna korelacija sa temperaturom vode. Istraživanje ove znanstveno zanemarene vrste će zasigurno biti nastavljeno sa ciljem boljeg razumijevanja i rasvjetljavanja njene geografske specifičnosti i varijabilnosti.

Ključne riječi: Fertilitet, morfologija, peš, rijeka Drina, rijeka Una 